\title{
Measurement of Floating Potential and Ion Concentration in Arc Plasma at Atmospheric Pressure
}

\section{K. Jha, L. N. Mishra and B. Narayan}

Journal of Nepal Physical Society

Volume 6, Issue 1, June 2020

ISSN: 2392-473X (Print), 2738-9537 (Online)

Editors:

Dr. Binod Adhikari

Dr. Manoj Kumar Yadav

Mr. Kiran Pudasainee

JNPS, 6 (1), 113-116 (2020)

DOI: http://doi.org/10.3126/jnphyssoc.v6i1.30559

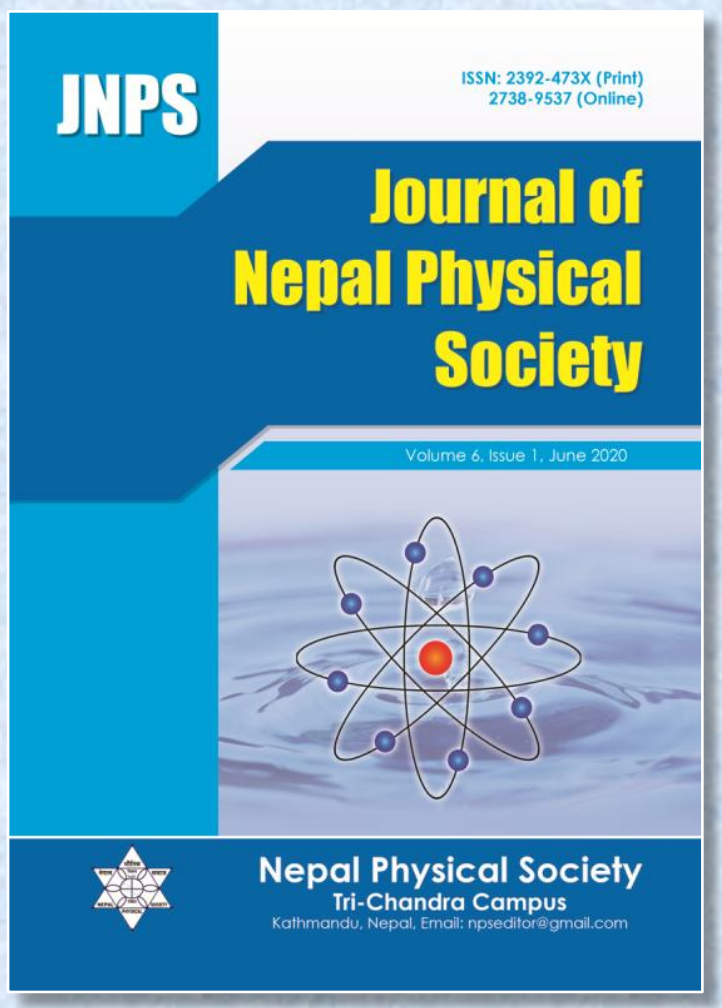

Published by:

Nepal Physical Society

P.O. Box: 2934

Tri-Chandra Campus

Kathmandu, Nepal

Email: npseditor@gmail.com 


\title{
Measurement of Floating Potential and Ion Concentration in Arc Plasma at Atmospheric Pressure
}

\author{
V. K. Jha ${ }^{1, *}$, L. N. Mishra ${ }^{2}$ and B. Narayan ${ }^{3}$ \\ ${ }^{1}$ Central Department of Physics, Kirtipur (Tribhuvan University), Kathmandu, Nepal \\ ${ }^{2}$ Patan Multiple Campus, Patandhoka, Lalitpur (Tribhuvan University, Kathmandu), Nepal \\ ${ }^{3}$ J. L. College, Hajipur, Vaishali (B. R. A. Bihar University, Muzaffarpur, Bihar), India \\ *Corresponding Email: jhav7050@gmail.com
}

Received: 15 Feb., 2020; Revised: 18 May, 2020; Accepted: 29 Jun., 2020

\begin{abstract}
Arc plasma is generated using low voltage dc power supply for the measurement of plasma parameters such as floating potential and ion-concentration in the plasma seeded with molybdenum. Langmuir moving probe is used in order to measure the probe current at different values of the dc potential applied on the probe. A graph is plotted between the probe current and the probe potential, based on data at the atmospheric pressure, using the experimental set up in the gas discharge chamber for the single probe method. The floating potential is calculated to be $32 \mathrm{~V}$, and the average ion-concentration to be $1.43-10^{16} \mathrm{~m}^{3}$.
\end{abstract}

Keywords: Langmuir Probe, Seeded arc plasma, Floating Potential, Ion- concentration.

\section{INTRODUCTION}

There is a rapidly growing interest for seeded arc plasma research which promises a wide range of industrial applications. A plasma arc operates on principles similar to an arc-welding machine, where an electrical arc is struck between two electrodes. The plasma arc is enclosed in a chamber. Waste material is fed into the chamber and the intense heat of the plasma breaks down organic molecules (such as oil, solvents, and paint) into their elemental atoms. In a carefully controlled process, these atoms recombine into harmless gases such as carbon dioxide. Solids such as glass and metals are melted to form materials, similar to hardened lava, in which toxic metals are encapsulated. With plasma arc technology there is no burning or incineration and no formation of ash. There are two main types of plasma arc processes known as plasma arc Melter and plasma torch.

Plasma arc Melter have a very high destruction efficiency. They are very robust and they can treat any waste with minimal or no pre-treatment; and they produce a stable waste form. The arc Melter use carbon electrodes to strike an arc in a bath of molten slag. The consumable carbon electrodes are continuously inserted into the chamber, eliminating the need to shut down for electrode replacement or maintenance. The high temperatures produced by the arc convert the organic waste into light organics and primary elements. The plasma arc can be used for organic and inorganic wastes. It is being studied for mixed radioactive waste treatment, because it separates the organic from the inorganic portion of the waste. It is also being studied to reduce explosive compounds and unexploded ordnance in place of traditional technologies, such as open burning and open detonation that produced toxic emissions and hazardous ash. It has also been used to thermally reduce asbestos to a slag that can be disposed of in a municipal landfill.

In plasma torch systems, an arc is struck between a copper electrode and either a bath of molten slag or another electrode of opposite polarity. As with plasma arc systems, plasma torch systems have very high destruction efficiency; they are very robust; and they can treat any waste or medium with minimal or no pre-treatment. The inorganic portion of the waste is retained in a stable, leachresistant slag. The air pollution control system is larger than for the plasma arc system, due to the need to stabilize torch gas.

Measurement of Plasma Parameters [1,2] is of immense importance for plasma processing [3-5]. 
It is also known as cold plasma of higher density, or low temperature plasma. However, its temperature is high enough to get the probe melted in a fraction of second when placed in it. The probe is, therefore, kept moving through the arc. Experimental data for the molybdenum seeded arc plasma is obtained at the atmospheric pressure using the experimental set up in the lowcost vacuum chamber [6]. Plasma parameters such as floating potential $\left(V_{\mathrm{F}}\right)$ and ionconcentration $\left(n_{i}\right)$ are calculated using Langmuir Probe [5, 7-11]. Single probe method is used in this study to measure these plasma parameters in the arc plasma, seeded with molybdenum.

\section{THEORY}

Basic equations $[12,13]$ for Langmuir probe (single probe method) are as follows:

$I_{\mathrm{e}}=\exp \tilde{\left(e V / k T_{\mathrm{e}}\right)}$

For $V=0, I_{\mathrm{e}}=\left(I_{\mathrm{e}}\right)_{\mathrm{r}}$ and so we can write

$n_{e}=\frac{\left(I_{\mathrm{e}}\right)_{\mathrm{r}}}{e A}\left(\frac{2 \pi m_{\mathrm{e}}}{k T_{\mathrm{e}}}\right)^{1 / 2}$

$n_{i}=\frac{2.5 I_{i}}{e A}\left(\frac{m_{i}}{2 k T_{e}}\right)^{1 / 2}$

Here $I_{\mathrm{e}}$ denotes the electrons current. $I_{i}$ is the current due to ions, $n_{\mathrm{e}}$ the electron-concentration, $\mathrm{n}_{i}$ the ion-concentration, $e$ the charge on each electron, $A$ the surface area of the probe, $k$ the Boltzmann's constant, $T_{\mathrm{e}}$ the electron's temperature, $m_{\mathrm{e}}$ the mass of an electron, $m_{i}$ the mass of an ion, and $V$ the probe potential.

Equation (1) is used for measuring the electron temperature $\left(T_{\mathrm{e}}\right)$ by drawing a tangent to the curve of $\ln I$ versus $V$ of the probe. Once the electron temperature is found, one can obtain the electron density using equation (2). The ionconcentration is found using equation (3) for a particular value of ion-current. After calculating various ion-concentrations for different values of ion-current, the average ion-concentration in the arc plasma can be determined. For a sufficiently negative probe potential, a stage comes when the drawn electron current just cancels the ion current. This takes place when $V=V_{\mathrm{s}}$. Here, $V_{\mathrm{s}}$ represents the space potential. Langmuir probe method is useful for study of a plasma of moderate density.

\section{EXPERIMENTAL SET UP}

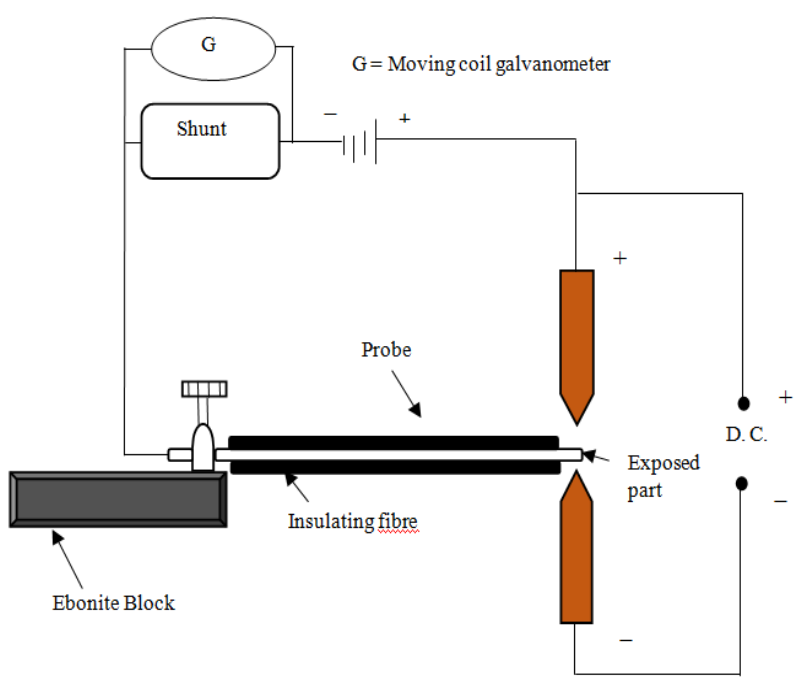

Figure 1: Schematic Diagram for Single Probe Method

Figure (1) shows the schematic diagram of the experimental set up for the single probe method in the low-cost chamber. A burning arc behaves like a dynamic plasma medium. The cylindrical probe wire of tungsten is supported on an ebonite base. The major portion of the probe is covered with insulating fiber leaving only a small part at its front uncovered (uninsulated). The probe is made to move through the arc.

\section{Table 1: Specifications of the Probe and Electrodes}

\begin{tabular}{|l|}
\hline Probe material: Platinum \\
\hline Length of the probe $=0.18 \mathrm{~mm}$ \\
\hline Average radius of the probe $=0.55 \mathrm{~mm}$ \\
\hline Surface area of the probe $(A)=1.57-10^{6} \mathrm{~m}^{2}$ \\
\hline Average diameter of the arc $=8.25-10^{3} \mathrm{~m}$ \\
\hline Average velocity of the probe $=20.0-10^{3} \mathrm{~mm} \mathrm{~s}^{1}$ \\
\hline Passage time of the probe through the arc $=0.46 \mathrm{~s}$ \\
\hline Diameter of copper-electrodes $=5.51 \mathrm{~mm}$ \\
\hline
\end{tabular}

\section{RESULTS AND DISCUSSION}

In the present experiment, diagnostics are performed using cylindrical Langmuir probe. Single-probe measurements are carried out in the region of molybdenum seeded arc plasma between two electrodes. 
Table 2: Variation of probe current with probe potential

\begin{tabular}{|c|c|}
\hline Probe Potential in volts $(\boldsymbol{V})$ & $\ln \boldsymbol{I}$ \\
\hline 0 & 7.798 \\
\hline-3 & 7.633 \\
\hline-6 & 7.565 \\
\hline-9 & 7.298 \\
\hline-12 & 6.129 \\
\hline-15 & 6.883 \\
\hline-18 & 6.665 \\
\hline-21 & 6.287 \\
\hline-24 & 5.883 \\
\hline-27 & 5.167 \\
\hline-30 & 1.889 \\
\hline-33 & -1.467 \\
\hline-36 & -2.954 \\
\hline-39 & -3.667 \\
\hline-42 & -3.789 \\
\hline-45 & -3.876 \\
\hline-48 & -3.933 \\
\hline-51 & -3.998 \\
\hline-54 & -3.987 \\
\hline-57 & -3.998 \\
\hline-60 & -3.998 \\
\hline
\end{tabular}

At large negative values of $V$ almost all the electrons are repelled, and we have an ion sheath and saturated ion current. There are two points of asymmetry between ion saturation and electron saturation due to their mass differences, which causes the disparity in the absolute magnitude of the currents. Since the ion and electron temperatures are usually unequal, and it turns out that sheath formation which is considerably different when the colder species are collected than that of when hotter species are collected.

Figure (2) shows the typical I-V characteristics of Langmuir probe in molybdenum sealed arc plasma where the lower portion of the curve represents ioncurrent saturation and that of the upper portion of the curve represents electron-current saturation.

In the present research work, the Langmuir probe is biased from 0 to $-60 \mathrm{~V}$ to drag ions so that its concentration can be characterized. In this technique, $I-V$ characteristic is depicted which is a fundamental part of diagnosis of plasma parameters such as electron temperature, electron density, ion temperature, ion density and so on. There is slow and non-uniform decrease in probe current as the probe voltage is changed from 0 to $-27 \mathrm{~V}$. From $-27 \mathrm{~V}$ to $-33 \mathrm{~V}$, the probe current rapidly decreases at uniform rate, as indicated by straight line part of the curve and the current becomes zero at $-32 \mathrm{~V}$ which is the floating potential of this arc plasma. The current decreases slowly with the change in probe voltage in the range from at $-36 \mathrm{~V}$ to at $-48 \mathrm{~V}$. The probe current becomes almost constant as the probe potential is changed in the range from $-50 \mathrm{~V}$ to $-60 \mathrm{~V}$. It is obvious from Fig. 2 that the floating potential in the arc plasma is found to be $-32 \mathrm{~V}$.

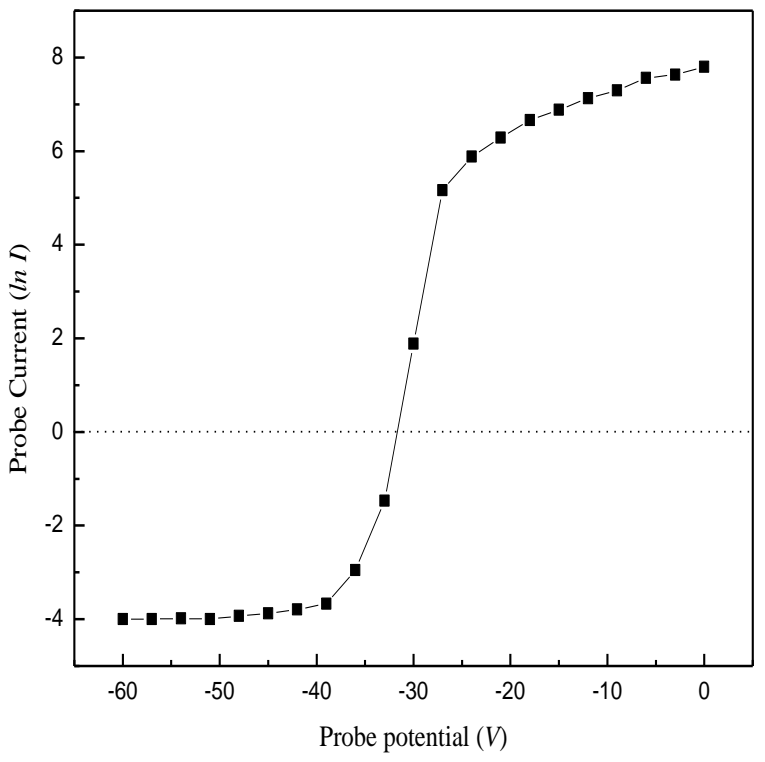

Figure 2: I-V Characteristics for single probe method in molybdenum -seeded arc plasma

Table 3: Calculation for Ion-concentration

\begin{tabular}{|c|c|}
\hline $\begin{array}{c}\text { Maximum Ion-Current } \\
\text { (micro-ampere) }\end{array}$ & $\begin{array}{c}\text { Maximum Ion- } \\
\text { Concentration (No. } \\
\text { of ions per cubic } \\
\text { meter) }\end{array}$ \\
\hline 3.998 & $\begin{array}{c}1.19-10^{16} \\
\text { (minimum ion- } \\
\text { concentration) }\end{array}$ \\
\hline
\end{tabular}

The maximum ion-concentration (Table 3 ) for ioncurrent of magnitude $3.998-\mathrm{A}$ is $1.19-10^{16} \mathrm{~m}^{3}$ and the average ion-concentration is $1.43-10^{16} \mathrm{~m}^{3}$ in seeded arc plasma at atmospheric pressure.

\section{CONCLUSIONS}

Langmuir single probe method is successfully used for measuring plasma parameters (floating potential and ion-concentration) in a molybdenum seeded arc plasma. This piece of study is expected to provide a new perspective for the industrial applications 
such as plasma processing, plasma torch of high efficiency, efficient arc lamps, etc.

\section{ACKNOWLEDGEMENTS}

The financial support for the present study by the University Grants Commission (UGC), Nepal, is appreciated. Authors are indebted to the Central Department of Physics (CDP), Tribhuvan University, for the valuable support. Keen interest taken both by Dr. Ghanshyam Thakur and Dr. Ajay Kumar Jha for the discussion related to the work is highly acknowledged.

\section{REFERENCES}

[1] Pulinets, M. S., Kirpichev, I. P. and Antonova, E. E. Variations in Plasma Parameters and Magnetic Field upon Magnetopause Crossing at the Main Phase Maximum of the Magnetic Storm of November 14, 2012. Geomagnetizm and Aeronomiy, 56, 673-681 (2016).

[2] Mishra, L. N., Shibata, K., Ito H., Yugami, N., Nishida, Y. Characteristics of electron Cyclotron resonance plasma Generated in a rectangular wave guide by high power Microwave. Review of Scientific Instruments, 75, 84-89 (2004).

[3] Conrads, H. and Schmidt, M. Plasma generation and plasma sources. Plasma Sources Science Technology, 9, 441-454 (2000).

[4] Ben Salem, D., Carton, O., Fakhouri, H., Pulpytel J. and Arefi $\square$ Khonsari, F. Deposition of water stable plasma polymerized acrylic acid/MBA organic coatings by atmospheric pressure air plasma jet. Plasma Processes and Polymers, 11, 269-278 (2014).

[5] Robert, B.L., and Brian, E. B. Recommended Practice for Use of Langmuir Probes in Electric Propulsion Testing. Journal of Propulsion and Power, 33, 566-581 (2017).
[6] Jha, V. K., Mishra, L. N. and Narayan B. Development of a Low-Cost Vacuum Chamber for the Experimental study of plasma parameters in a seeded arc plasma. International Journal of Latest Engineering and Management Research, 3, 13-18 (2018).

[7] Dejarnac, R. P., Stangeby, C., Goldston, R. J., Gauthier, E., Horacek, J., Hron, M., Kocan, M., Komm, M., Panek, R, Pitts R. A. and Vondracek, P. Understanding narrow SOL power flux component in COMPASS limiter plasmas by use of Langmuir Probes. Journal of Nuclear Materials, 463, 381-384 (2015).

[8] Sinha, R. P., Prasad, B. M. K., Bhagat, M. and Narayan, B. A study of Arc Plasma Using a Moving Langmuir Probe. Indian Journal of Pure \& Applied Physics, 26: 20-21 (1988).

[9] Rakhimova, T. V., Braginsky, O. V., Ivanov, V. V., Kim, T. K., Kong, J. T., Kovalev, A. S., Lopaev, D. V., Mankelevich, Y. A., Proshina, O. V. and Vasilieva A. N. Experimental and Theoretical Study of RF Plasma at Low and High Frequency. IEEE Transaction on Plasma Science, 34, 867-877 (2006).

[10] Doggett, B., and Lunney, J.G., Langmuir probe characterization of laser ablation plasmas, Journal of Applied Physics, 105, 033306 (2009).

[11] Mishra, L. N., Shibata, K., Ito H., Yugami, N., Nishida, Y. Pulsed Corona Discharge as a Source of Hydrogen and Carbon Nanotube Production, IEEE Transaction on Plasma Science, 32, 1727 1733 (2004).

[12] Boyd, T. J. M. and Sanderson, J. J. Plasma dynamics, Thomas Nelson Sons, London, UK (1969).

[13] Thakur, G., Khanal, R., \& Narayan, B. Characterization of Arc Plasma by Movable Single and Double Langmuir Probes, Fusion Science and Technology, 75, 324-329 (2019). 